\title{
Quality improvement in resident education: a pilot project to mitigate metabolic side effects from atypical antipsychotic medications in youth
}

\author{
Jessica Jeffrey
}

UCLA, USA

\begin{abstract}
This resident physician-led quality improvement project was conducted with aims to improve the health of youth prescribed atypical antipsychotic medications by increasing physician monitoring for metabolic side effects, while simultaneously educating trainees in quality improvement methodology.
\end{abstract}

The plan, do, study, act quality improvement framework was utilized. Baseline metabolic monitoring rates of patients prescribed atypical antipsychotic medications in the two psychiatry resident outpatient clinics were obtained. Rates were stratified based on time on medication ( $<1$ year, $\geq 1$ year) and parameter monitored. Metabolic monitoring rates subsequent to targeted changes were obtained. Problem solving with residents revealed barriers to monitoring, such as limited awareness of specific guideline recommendations and lack of convenient access to medical equipment (calibrated scales). Residents received education about atypical antipsychotic monitoring guidelines and side effect treatment. Residents were provided with calibrated scales. Atypical antipsychotic monitoring templates were introduced. Online surveys using were conducted to determine self-reported baseline-monitoring rates and comfort with guidelines following targeted change.

The baseline metabolic monitoring rates of patients prescribed atypical antipsychotic medications was $9 \%$ (range: 0 to $17.6 \%$ ) for youth in their first year taking an atypical antipsychotic medication and 58.9\% (range: $29 \%$ to $100 \%$ ) in subsequent years on medication. The results of relatively easy changes resulted in modest improvement in monitoring rates. The metabolic monitoring rate of a patient initiated on an atypical antipsychotic medication was $29 \%$ after targeted quality improvement measures were employed. Following quality improvement changes, residents reported increased knowledge about guidelines and increased monitoring for side effects. Use of a standardized data collection instrument to track monitoring of patients increased from $0 \%$ to $70 \%$ (range: $30 \%$ to $90 \%$ ).

Quality improvement projects provide an avenue with which to improve atypical antipsychotic monitoring rates. Through active participation in quality improvement projects, psychiatry residents may be taught to employ quality improvement methodology.

\section{Problem}

Atypical antipsychotic medication use in children is rapidly increasing in the United States, despite significant potential side effects, with long-term health implications.[1,2] According to the Center for Medicaid Services Data for 50 states and the District of Columbia antipsychotic use increased by $62 \%$ from 2002 through 2007 , reaching 354,000 or $2.4 \%$ of youth by 2007.[1] Atypical antipsychotic medications are associated with several established cardiometabolic side effects, such as weight gain, dyslipidemia (including increased low-density lipoprotein (LDL), increased triglycerides, decreased high-density lipoprotein (HDL)) hyperglycemia, and type-2 diabetes mellitus.[2] Children and adolescents exposed to these cardiometabolic side effects from atypical antipsychotics are at risk for negative health outcomes both as youth and adults.

Given the seriousness of side effects associated with these medications, guidelines have been introduced to assist with monitoring and treatment of side effects. In general, these guidelines recommend monitoring of fasting lipids and glucose, height, weight, blood pressure, and waist circumference. Despite the establishment of these guidelines, recommendations are often not translated into clinical practice and little research exists regarding ways to improve metabolic screening rates. Systematic monitoring of atypical antipsychotic medication cardiometabolic side effects is an important skill to begin adopting during residency training to prevent negative health outcomes in patients.

\section{Background}

Individuals treated with atypical antipsychotic medications during critical periods of development may experience long-lasting negative health effects.[3] Some children remain on antipsychotic medications for extended periods of time, thus increasing exposure to these medications during critical periods of development. As children, those who are overweight have increased rates of metabolic syndrome[4] and type 2 diabetes.[5] Another study reported children who are overweight have increased rates of carotid intimal media thickening, which is a risk factor for having a cerebrovascular accident.[6] In addition to having negative health outcomes as children, Juonala et al (2005)[7] revealed obesity, high LDL, and low HDL in children are associated with decreased carotid artery elasticity in adulthood; decreased carotid artery elasticity is also a risk factor for having a cerebrovascular accident. A prospective cohort study of 2,195 children followed for 21 years in 


\section{BMJ Quality Improvement Reports}

Finland demonstrated childhood obesity, high triglycerides, high insulin, and high CRP are risk factors for adult metabolic syndrome.[8]

It is important for residents to feel empowered to undertake quality improvement (QI) endeavors, such as improving side effect monitoring of medications in order to improve clinical care provided to their patients. Quality improvement was included as a required core competency in systems-based care education by the United States Accreditation Council for Graduate Medical Education (ACGME) in July 2011[9,10]; however, there is little guidance on how best to integrate these ACGME guidelines within resident training. Furthermore, there are few examples as to how best to integrate resident physician-led QI programs into health systems managed by permanent staff. We describe an example of a QI project undertaken by child psychiatry residents within an outpatient clinical setting to address metabolic side effects related to atypical antipsychotic medications in children and adolescents.

\section{Baseline measurement}

This QI project occurred within two child psychiatry outpatient clinics at a large academic medical center in the United States. These clinics are staffed by six resident physicians, who all participated in the project. The clinics treat patients from ages 5 to 25 and there are approximately 400 patients enrolled in the clinics.

Resident physicians, medical school graduates who receiving training in a specialized area, reviewed practice guidelines for monitoring atypical antipsychotic medication side effects. The Canadian Alliance for Monitoring Effectiveness and Safety of Antipsychotics in Children (CAMESA) Guidelines (2011) were chosen for this project due to their comprehensiveness, availability of a free excel monitoring template for each atypical antipsychotic based on CAMESA guidelines and accessibility (for more information on the CAMESA tool, see http://camesaguideline.org for details and free tool).

The Consensus Development Conference on Antipsychotic Drugs and Obesity and Diabetes (2004) was also considered for this project,[13] but when compared to the CAMESA guidelines, we determined that the CAMESA guidelines appeared more comprehensive, as this guideline recommends monitoring for side effects at a greater number of time intervals, and specific to monitoring for metabolic side effects in children (table 1). Monitoring beyond year one was informed by the American Academy of Child and Adolescent Psychiatry Practice Parameter for the Assessment and Treatment of Children and Adolescents with Schizophrenia,[14] as CAMESA guidelines do not provide a formal recommendation beyond year one. After completion of the first year on an antipsychotic medication, it is recommended monitoring of metabolic parameters occur every six months, with more frequent monitoring as clinically indicated.[14]

Based on the aim of this project to improve compliance with guidelines, several metrics were identified by the resident project leader and faculty mentor, including both outcome and process metrics. Residents then engaged in a discussion about these metrics and selected the ones to be used for this QI effort. Metrics were collected at baseline (month one) and follow-up (month seven).

Process metrics included measurement of change in trainee knowledge of monitoring guidelines and how to use a standardized instrument to monitor cardiometabolic side effects as well as selfreported facilitators and barriers to monitoring. An eight item online anonymous survey administered at pre and post QI activities assessed trainee self-report of knowledge of guidelines for monitoring the use of atypical antipsychotic medications in adolescents and children. Items were rated on a Likert scale of 1 to 5 ( 1 unaware of guidelines, 3 somewhat knowledgeable of guidelines, 5 very knowledgeable of guidelines).

For the primary outcome metric, residents decided to measure the monitoring rate of metabolic side effects for patients on atypical antipsychotic medications. Given the time frame of seven months in which to implement this QI activity, health outcomes such as reduction in lipid profile were not feasible. For baseline measures, residents conducted a chart review of up to five of their own patients, all of whom had been transferred to them at the beginning of the academic year. Follow up chart review was conducted at month seven of all patients initiated on an atypical antipsychotic within that time period. Chart review eligibility criteria included patients who were: 1 ) $<18$ years of age; 2 ) currently prescribed an atypical antipsychotic medication; and 3 ) taking that medication for at least six months prior to the review. A total of 13 cases were reviewed, with an average of 2.2 charts per resident reviewed; less than five charts per resident reviewed indicated that the resident's caseload had less than five patients prescribed atypical antipsychotic medication. The resident that completed a chart review of five patients had eight patients taking atypical antipsychotic medications. Therefore, there were a total of 16 patients identified as taking antipsychotic medications within their combined care in the outpatient clinic setting.

Residents reviewed their own charts for compliance with a total of 7 CAMESA cardiometabolic parameters (height; weight; metabolic labs, including fasting plasma glucose or fasting insulin; fasting total cholesterol; fasting low-density lipoprotein (LDL); fasting highdensity lipoprotein (HDL); fasting triglycerides) using the CAMESA excel monitoring template. Residents then reported de-identified monitoring results to the resident project leader. Metabolic monitoring rates were calculated by utilizing the following formula: number of parameter points monitored divided by the number of parameter points recommended for monitoring. A parameter point is defined as a data point recommended for collection according to CAMESA guidelines. For instance, at baseline the number of parameter points recommended for monitoring is seven (height; weight; metabolic labs including fasting plasma glucose or fasting insulin; fasting total cholesterol; fasting LDL; fasting HDL; fasting triglycerides) and at one month the number of parameter points recommended for monitoring is 2 (height; weight). The number of parameter points recommended for monitoring differed based on whether a patient had been taking a medication for $<1$ year or $\geq 1$ year (34 and 14 recommended parameters, respectively). 
BMJ Quality Improvement Reports

The process metrics indicated low overall systematic monitoring of metabolic side effects in patients treated with atypical antipsychotic medications. None of the residents reported using a standardized monitoring tracking template prior to this QI project, and the average rating for knowledge about guidelines was 3.2 (range: 2 to 4).

The average baseline metabolic monitoring rates of patients prescribed atypical antipsychotic medications was $9 \%$ (range: $0-17.6 \% ; n=5$ ) for children and adolescents in their first year taking an atypical antipsychotic medication and 58.9\% (range: 29\%-100\%; $\mathrm{n}=8$ ) in subsequent years on the medication (table 2). Rates of monitoring were also classified according to the type of parameter monitored. For children and adolescents in their first year taking an atypical antipsychotic medication, monitoring rates averaged $29 \%$ for height, $49 \%$ for weight, and $60 \%$ for metabolic parameters. The monitoring rate for those taking antipsychotic medications for greater than one year averaged $44 \%$ for height, $69 \%$ for weight, and $56 \%$ for metabolic parameters. Further examination of trends in monitoring revealed two instances of patients who received blood draws in their first year taking antipsychotic medications but did not have all recommended parameters recorded; plasma glucose was the only parameter measured, despite recommendations to monitor fasting lipids as well. For those taking atypical antipsychotic medications in subsequent years, five patients received blood draws but did not have all recommended parameters recorded; in these instances fasting lipids were measured but plasma glucose was not measured.

See supplementary file: ds5389.docx - "Comparison of CAMESA AND CC Guidelines"

\section{Design}

This QI project was conducted over an eight month period in two child and adolescent psychiatry general outpatient training clinics at an academic institution during the 2013-2014 academic year. The QI project was conceived and led by one child psychiatry resident (the resident project leader) with supervision from a child psychiatry faculty mentor and support from the child and adolescent faculty clinic supervisors. Six second-year child and adolescent psychiatry residents, including the resident project leader, participated in the QI project. An Institutional Review Board (IRB) exemption was granted by the IRB of a large academic medical center.

The plan, do, study, act (PDSA) framework guided the development of this resident-led QI project.[11] This project used a participatory process with the clinic residents and faculty to set the goals and targets for improvement. Following an educational session on monitoring guidelines for antipsychotics in children, the residents determined that the aim of the project would be to obtain $80 \%$ compliance with recommended metabolic monitoring rates of atypical antipsychotic medications by June 2014. Although the residents recognized that this goal might be ambitious, they felt that given the potential long-term implications of these side effects, a high standard for compliance was an important goal.

\section{Strategy}

The resident project leader and faculty mentor developed a causal factors analysis based on feedback from clinic discussions with residents and clinic faculty, which revealed several potential contributors to low atypical antipsychotic medication monitoring rates (figure 1). These included environmental factors, poor access to materials to assist with monitoring, and human factors such as motivational and skills/knowledge factors. Residents are required to obtain vitals for their patients in outpatient clinics, as the clinics do not employ staff or utilize volunteers to obtain vitals. Residents expressed that it was difficult for them to obtain vitals, conduct the appointment, and present the patient to the attending physician within a 30 minute follow up appointment. Given health system financial constraints, residents are required to collect vital signs and maintain 30 minute follow up appointment times; thus, this was not determined to be an easy change to focus on for initial improvement of antipsychotic monitoring rates.

Residents also expressed frustration with the fact that at times when they ordered labs for their patients, their patients did not present to the laboratory to have their labs drawn. The group discussed this may be due to several issues, including that the laboratory is located in another building. Additionally, patients often do not return to complete fasting labs. Several issues may impact whether a child returns for a laboratory draw, such as their parents' ability to take time off work, transportation difficulties, or child care issues for other children in the family. Given the complexity of these issues, this was not determined to be a straightforward change to focus on for initial improvement in monitoring rates.

Putative causal factors analysis also revealed residents lacked convenient access to medical equipment in order to monitor for side effects to atypical antipsychotic medications. Specifically, residents stated they had scales that were placed directly on carpet, thus rendering readings from the scale inaccurate measures of weight. They also reported having pediatric-size blood pressure cuffs in their offices and stated they also required adult-size blood pressure cuffs for blood pressure monitoring of adolescent patients. The residents stated they did not use a standardized data collection instrument to monitor for side effects. Residents stated a standardized method of collecting data would make it easier to remember to monitor for side effects at the recommended intervals. Potential benefits of building metabolic side effect monitoring parameters into the electronic health record (EHR) were enthusiastically discussed; however, discussion with technology staff revealed this would not be feasible for approximately one year given EHR systems updates. Given the fact that changes in materials are relatively straightforward changes to make, this was a focus of the QI endeavor. To address barriers related to materials factors, residents were provided with boards to place under scales in their offices so as to increase accuracy of measurement. CAMESA atypical antipsychotic monitoring templates were also introduced.

Human factors postulated by residents to act as barriers to monitoring for side effects to antipsychotic medications included motivational and skills/knowledge factors. Side effects to atypical 
antipsychotic medications may develop over years, thus making the side effects less salient. Also, residents suggested that financial or legal incentives to comply with monitoring activities would increase motivation. Residents were unaware of legal cases involving monitoring for side effects, which would potentially increase salience of this issue and motivation for monitoring. Other human factors included educational factors, such as limited awareness of specific guideline recommendations. Given the practicality of potential interventions, skills/knowledge factors were addressed during this QI project. To address skills/knowledge barriers, fellows received education about atypical antipsychotic monitoring guidelines and treatment of side effects, using the CAMESA Guidelines [12] and the Practice Parameter for the Assessment and Treatment of Children and Adolescents with Schizophrenia.[14] CAMESA guidelines for management of metabolic side effects were also discussed.

\section{Results}

Self-reported use of a standardized data collection instrument to track monitoring of patients increased from $0 \%$ at baseline to $70 \%$ at follow-up (range: $30 \%$ to $90 \%$ ). Residents rated their knowledge of guidelines increased to an average of 4.2 (range 4 to 5). Comments made during the survey revealed the addition of a standardized data collection instrument "provide[s] clear concise reminders of what data need to be monitored at which visit" and ultimately "decrease[s] long-term morbidity of chronic medical conditions". One respondent suggested incorporation of a standardized data collection instrument into the electronic medical record for further ease of data collection.

There was only one patient initiated on an atypical antipsychotic medication in the clinics over the course of the project. The metabolic monitoring rate of the patient $(n=1)$ initiated on an atypical antipsychotic medication in the clinic was $29 \%$ after QI interventions were employed. This patient received height and weight monitoring, however labs were not obtained. The self-reported placement of boards under the scales in each fellow's office, in order allow each scale to be calibrated correctly, increased from $14 \%$ to $100 \%$ in each office.

See supplementary file: ds5390.docx - "Putative Causal Factors Analysis"

\section{Lessons and limitations}

QI projects consist of systematic and continuous actions designed to lead to improvements in patient health. QI frameworks may be utilized to address salient issues, such as low adherence to monitoring for cardiometabolic side effects associated with use of atypical antipsychotic medications. Little research exists regarding ways to improve metabolic screening rates, and this resident-led child and adolescent psychiatry atypical antipsychotic metabolic side effect monitoring project aimed to improve metabolic screening rates of youth prescribed atypical antipsychotic medications in two outpatient clinics.
The U.S. ACGME recently included QI as core competency in systems-based care education, $[9,10]$ yet despite the presence of this relatively new requirement there is minimal guidance regarding how best to integrate these guidelines within resident training. This QI project was resident-led, with faculty supervision and guidance, and involved the participation of six residents in a child and adolescent psychiatry training program. The project provides a useful illustration of ways a training program might incorporate QI into the curriculum. Although this project was conducted within the U.S., practical experiences using quality improvement techniques within training will benefit resident physicians in both the U.S. and abroad. The results of this QI project demonstrate the modest level of improvement that may be expected with relatively simple educational and materials changes in an outpatient clinic environment.

Group discussion with residents revealed several barriers to monitoring for side effects to atypical antipsychotic medications that were not readily apparent. The use of a putative causal factors analysis allowed for consolidation and organization of information collected. Given the feasibility of potential interventions revealed in the putative causal factors analysis (environmental factors, poor access to materials to assist with monitoring, and human factors such as motivational and skills/knowledge factors), materials and skills/knowledge factors were addressed during this QI project.

Despite increased access to medical equipment (calibrated scales) to allow for greater ease of monitoring, education about CAMESA guidelines and American Academy of Child and Adolescent Psychiatry Practice Parameter for the Assessment and Treatment of Children and Adolescents with Schizophrenia monitoring guidelines and introduction of the CAMESA excel standardized data collection instrument, the follow up chart review revealed only a modest improvement in the monitoring rate (increase from $9 \%$ to $29 \%$ ) of one patient initiated on an atypical antipsychotic medication during the eight-month project period. The aim of the project was to obtain $80 \%$ compliance with recommended metabolic monitoring rates of atypical antipsychotic medications by June 2014. This aim, set by the residents themselves as a motivating factor, was overambitious given the baseline monitoring rates of $9 \%$ for patients in their first year on atypical antipsychotic medications and $58.9 \%$ for those in subsequent years.

Although the self-reported use of a standardized data collection instrument to track monitoring of patients increased from $0 \%$ to $70 \%$ (range: $30 \%$ to $90 \%$ ), this also did not meet the aim of obtaining $80 \%$ average compliance with monitoring rates by June 2014 . Ratings of self-reported knowledge of monitoring guidelines increased, as did access to calibrated scales in fellows' offices. In addition to increased access to equipment, workflow was reported to improve through introduction of a standardized instrument to monitor for cardiometabolic side effects.

Although the small sample size precludes statistical analysis, it is interesting to note baseline rates of those patients in their first year taking an atypical antipsychotic medication were lower for height ( $29 \%$ vs $44 \%$ ) and weight ( $49 \%$ vs. $69 \%$ ), than patients in subsequent years on antipsychotic medication; however, rates of 
monitoring for metabolic parameters were almost equivalent $(60 \%$ vs $56 \%$ ). This may be due to the fact the first year of monitoring recommends more parameters are monitored (height and weight at baseline, one month, two months, three months, six months, nine months, one year) as compared with subsequent years on the medication (every six months). Despite the rigor of monitoring recommendations in the first year as compared with the subsequent year, monitoring of metabolic parameters was similar. Closer examination of baseline metabolic monitoring rates revealed missed opportunities for monitoring of metabolic parameters, as in seven instances (two instances for patients in their first year taking antipsychotic medications and five for those in subsequent years) blood draws obtained some, but not all, recommended metabolic screening measures. These results suggest knowledge of recommended monitoring parameters may improve monitoring rates, as these patients received laboratory monitoring; however, several labs were not ordered per guideline recommendations. Given the short limited timeframe of this project, only one patient was initiated on an atypical antipsychotic medication during the project period and this child did not receive metabolic monitoring.

There are several limitations to this QI educational pilot project. As mentioned above, given the small sample size of this QI project precludes statistical analysis. Additionally, outcome measures included resident's report of use of the CAMESA excel monitoring template and self-reported knowledge of monitoring guidelines. Future QI projects may investigate objective measures of resident knowledge of monitoring guidelines. Also, given the eight month time frame of this project there were limited opportunities to examine follow up monitoring rates since only one patient was initiated on an atypical antipsychotic medication in the clinics during this time. Despite these limitations, this project provides a useful example of the kinds of relatively simple changes that may be addressed in a QI project and the expected outcomes from such changes.

Given the limited improvement in monitoring rates evident in this project, there are still further changes that may be made to the system to increase adherence to monitoring for metabolic side effects. The putative causal factors analysis suggests other potential changes to the health system, such as investigating options for incorporation of a standardized data collection instrument into the electronic health record. This potential QI change was also mentioned by trainees in the online survey and during group discussion as a way to streamline workflow. Automated reminders built into the electronic health record regarding which parameters should be monitored at specific time points may further increase rates. Baseline monitoring rates revealed missed opportunities to obtain recommended metabolic labs. Other systematic improvements may include presence of a staff member to obtain vitals, as the putative causal factors analysis revealed limited appointment time as a constraining factor to obtaining vital signs such as height and weight. Further QI projects may test these changes on the desired aim of improving monitoring rates.

\section{Conclusion}

Through introduction to QI concepts and participation in a QI project child psychiatry residents learned valuable concepts which may be utilized in various clinical situations to promote health. QI is an important part of the culture of graduate medical training in the Child and Adolescent Psychiatry Program, preparing trainees to critically look at their environments and to embrace the testing of small changes, with the aim to work towards improvements in patient health. Given the well-established side effects of atypical antipsychotic medications, it is crucial physicians-in-training comply with recommended monitoring guidelines and address any side effects as they arise. QI methodology may be used to address this problem and improve patient care. Through utilizing QI projects, the health of youth may be improved.

\section{References}

1. Matone M, Localio R, Huang YS, et al: The relationship between mental health diagnosis and treatment with secondgeneration antipsychotics over time: a national study of U.S. Medicaid-enrolled children. Health Services Res 2012; 47;1836-60.

2. Dulcan, Mina, Editor. Dulcan's Textbook of Child and Adolescent Psychiatry 1st Edition. Washington, DC: American Psychiatric Publishing; 2010.

3. De Hert M, Dobbelare M, Sheridan EM, et al: Metabolic and endocrine adverse effects of second-generation antipsychotics in children and adolescents: A systematic review of randomized, placebo-controlled trials and guidelines for clinical practice. Eur Psychiat 2011; 26:144-58.

4. Weiss R, Dziura J, Burgert TS, et al: Obesity and the metabolic syndrome in children and adolescents. New Engl J Med 2004; 350:2362-74.

5. Maayan L, Correll CU: Weight Gain and Metabolic Risks Associated with Antipsychotic Medications in Children and Adolescents. J Child Adol Psychop 2011;21:517-35.

6. Urbina EM, Kimball TR, McCoy CE, et al: Youth with obesity and obesity-related type 2 diabetes mellitus demonstrate abnormalities in carotid structure and function. Circulation 2009;119:2913-9.

7. Juonala M, Jarvisalo M, Makl-Torkko N, et al: Risk factors identified in childhood and decreased carotid artery elasticity in adulthood. Circulation 2005;112:1486-93.

8. Mattsson N, Ronnemaa T, Juonala M, et al: Childhood predictors of the metabolic syndrome in adulthood. The cardiovascular risk in young finns study. Ann Med 2008; 40:542-2.

9. Krupa C. Residency programs scramble to adopt changes. In: American Medical News. 2011. http://www.amednews.com/article/20110711/profession/307 119948/4/. Accessed July 11, 2011.

10. Benzer JK, Bauer M, Charna M, Topor D, Dickey C: Resident/Faculty Collaboration for Systems-Based Quality Improvement. Acad Psychiat. 2013; 37:6: 433-5.

11. Langley G, Moen R, Nolan K, et al: The Improvement Guide: A Practical Approach to Enhancing Organizational Performance. 2nd ed. San Francisco, CA: Jossey-Bass; 2009. 


\section{BMJ Quality Improvement Reports}

12. Pringsheim, T, Doja A, Belanger S, et al: Evidence-Based Recommendations for Monitoring of Safety of Second Generation Antipsychotics in Children and Youth. J Canadian Acad Child Adol Psychiat 2011; 20:218-33.

13. Consensus development conference on antipsychotic drugs and obesity and diabetes. Diabetes Care 2004;27:596-601.

14. McClellan J, Stock S. American Academy of Child and Adolescent Psychiatry Practice Parameter for the Assessment and Treatment of Children and Adolescents with Schizophrenia. J Canadian Acad Child Adol Psychiat. 2013; 52:976-90.

\section{Declaration of interests}

Nothing to declare.

\section{Acknowledgements}

This quality improvement project would not have been possible without the support of many people. I would like to express gratitude to my mentor, Dr Sheryl Kataoka who offered invaluable support and guidance. I would also like to thank the child and adolescent psychiatry trainees at UCLA for their assistance with this quality improvement project. Lastly, I would like to thank $\mathrm{Dr}$ Christoph Correll for inspiring this project.

\section{Ethical approval}

An institutional review board (IRB) exemption was granted by the IRB of a large academic medical center. 